\title{
Staying in the Flow with Zoomable User Interfaces
}

\author{
Xerox Palo Alto Research Center \\ 3333 Coyote Hill Rd \\ Palo Alto, CA 94304 USA \\ +1 650-812-4661 \\ good@parc.xerox.com
}

\section{Lance Good}

\author{
Human-Computer Interaction Lab \\ Computer Science Department \\ University of Maryland \\ College Park, MD 20742 USA \\ goodness@cs.umd.edu
}

\section{ABSTRACT}

This research aims to investigate a collection of interactions in 2D workspaces with the goal of helping users stay in the flow of their activity. These interactions will be explored in the context of two software tools designed to support information work. The first tool, Niagara, addresses the early phases of this work that involve organization and synthesis. CounterPoint, the second tool, targets the later stages of this work that concern the authoring, delivery, and understanding of presentations.

\section{Keywords}

Interaction techniques, staying in the flow, Zoomable User Interfaces (ZUIs), sensemaking, slide presentations.

\section{INTRODUCTION}

Previous research suggests several benefits of 2D spaces for representing non-spatial information in user interfaces. These spaces can exploit human memory for spatial location [4], encourage serendipity [1] and improvisation [4], portray hierarchical structures [4][5], and support the ambiguities of an organization task [5]. However, many of the basic interactions in these spaces, such as the authoring and navigation of non-spatial data, have not been systematically studied.

My work explores interaction techniques in these environments to help users stay in "the flow" of common information-centric tasks. "The flow" has been used in the popular psychology literature to describe the aspects of an activity that make it enjoyable and motivational [3]. My work focuses on the flow at a lower level, becoming evident in more traditional software usability metrics such as task completion time and subjective satisfaction. The principle behind staying in the flow is to reduce the complexity and interruptions of a tool to the point where it becomes a natural extension of the user. When this is achieved, users are free to focus on their task without being conscious of the interface.

The optimal interaction techniques for staying in the flow in many cases will depend heavily on the user's task. For instance, during the early stages of an organization task, the user may not require precision in arranging objects, making certain automated interactions appropriate. However, these

Copyright is held by the author/owner(s).

CHI 2002, April 20-25, 2002, Minneapolis, Minnesota, USA. ACM 1-58113-454-1/02/0004. same arrangements are likely to call for more precise control when presentation is the primary concern.

To understand the extent to which these techniques can be generalized across tasks, I am implementing two different tools targeted at different information-based tasks. The first tool, Niagara, explores the early stages of information synthesis, where collections of unorganized information are read, written, classified, arranged, annotated, processed, and summarized. We describe this task as sensemaking because it involves building an understanding, or making sense, of a collection of information. To support staying in the flow of the sensemaking task, Niagara will be used to explore different interaction techniques for each of the operations mentioned above.

The second tool, CounterPoint [4], deals with the later stages of the information process where organized information is laid out and presented to others. This presentation task has three components including authoring spatial layouts, creating scripted paths through the presentation, and actually delivering the presentation. Accordingly, CounterPoint will be used to explore interactions for staying in the flow of each of these three tasks.

\section{RELATED WORK}

Perhaps because of the familiarity of traditional 2D media such as paper and chalkboards, there are innumerable software systems that make use of 2D space for representing non-spatial information. These include tools for such tasks as drawing, creating node-link graphs, organizing information, and authoring presentations. PowerPoint [6] is one such tool that is used for a variety of tasks in this domain.

Alternately, a number of systems have introduced general techniques for interacting with non-spatial data in 2D spaces. Jazz is one such system that provides tools for interacting with Zoomable User Interfaces [2]. Although these techniques provide a general foundation, they have not been applied and empirically validated for staying in the flow of many information-centric tasks.

A number of research systems have also been developed to explore the use of 2D space for specific information tasks. The systems most closely related to my proposed research are those described as "spatial hypertext" [5]. However, while these systems address tasks relevant to my research, they have not traditionally focused on interaction techniques but rather on structure and representations. 
RESEARCH PROPOSAL

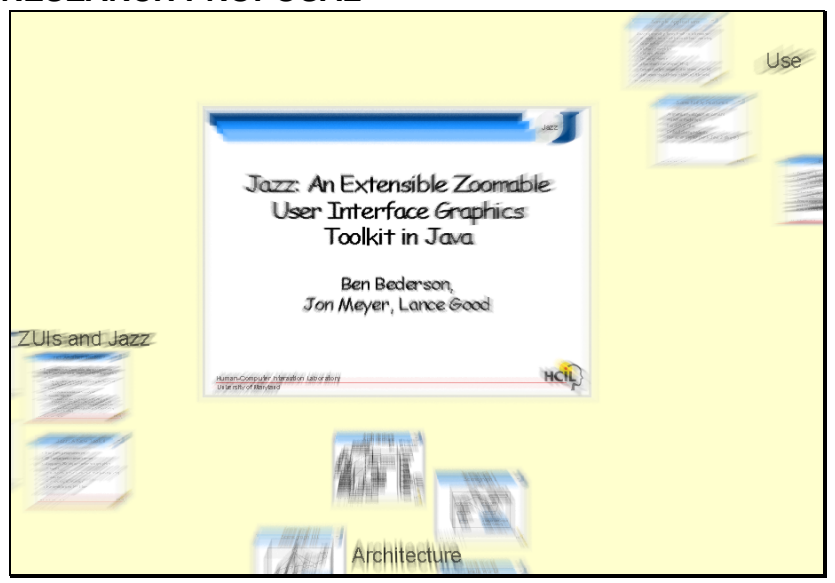

Figure 1. CounterPoint offers smoothly animated transitions through the presentation structure to help the audience stay in the flow of the presentation. This figure simulates zooming in on the title slide in a presentation on Jazz [2].

My proposal involves the development of two tools to support different information-based tasks. Yet at a more abstract level these tasks can be thought of as two components of a single process. This process begins with a potentially unorganized collection of knowledge, such as ideas, facts, or documents, and ultimately concludes with a polished slide presentation.

For the sensemaking component of this process, Niagara will serve as the platform for exploring techniques for staying in the flow. To inform the design of these interaction techniques, one of the early goals of my proposal is to develop a tool-independent model of the sensemaking process compiled from actual sensemaking sessions performed using existing physical and virtual tools. This model will serve as a guide to developing improved interaction techniques, as it will indicate the cost and frequency of the different sensemaking operations.

Once a promising interaction technique has been identified, I intend to determine its utility for staying in the flow by comparing user performance on a sensemaking task using versions of Niagara with and without the specified interaction. Consequently, the success of this portion of my proposal depends on finding a reasonable size set of promising interaction techniques.

Early experience and user testing with Niagara has revealed several critical tradeoffs in the design of these interactions for 2D spaces. The most salient of these tradeoffs include balancing spatial awareness against structural awareness and balancing automated organizational support against predictability and distraction.

The second part of my proposal will focus on CounterPoint as the medium of exploration. CounterPoint is intended to support both the authoring and delivery of zooming presentations. Because there are no tools dedicated to these tasks, my work with CounterPoint thus far has focused on developing a reference implementation from which alternate interaction techniques could be debated.

Perhaps the most fundamental question surrounding zooming presentations is whether they are more effective than traditional presentations. Consequently, my first CounterPoint study will assess whether CounterPoint presentations help the audience stay in the flow of the presentation. For this study, I intend to compare audience understanding, recall, and satisfaction with CounterPoint presentations versus traditional software presentations.

As work on both tools continues, I expect that interaction techniques from Niagara will often inform the design of CounterPoint and vice versa. In fact, a number of interactions have already been identified as common to both tools. These include techniques for improving awareness of object locations, defining containment within spatial hierarchical structures, coordinating multiple views, providing fluid navigation, interacting with automated object positioning, and defining scripted paths.

Lastly, one of the ultimate goals of my research is to develop a deeper understanding of the significance of the flow. Although it is frequently reflected in traditional usability metrics, the flow is often overlooked when it does not directly improve short-term completion times or error rates. Through my research I plan to identify tasks where the flow is especially important and characterize the flow's short and long-term effects on these traditional metrics.

\section{ACKNOWLEDGMENTS}

This work has been funded by DARPA and Xerox PARC. I would like to offer special thanks to my advisor, Ben Bederson, and lab manager, Mark Stefik, for their guidance and encouragement. I also thank Patrick Baudisch for help with images and constructive comments on this paper.

\section{REFERENCES}

1. Bederson, B. B. (2001). PhotoMesa: A Zoomable Image Browser Using Quantum Treemaps and Bubblemaps. UIST 2001, ACM Symposium on User Interface Software and Technology, CHI Letters, 3(2).

2. Bederson, B. B., Meyer, J., \& Good, L. (2000). Jazz: An Extensible Zoomable User Interface Graphics Toolkit in Java. UIST 2000, ACM Symposium on User Interface Software and Technology, CHI Letters, 2(2), pp. 171-180.

3. Csikszentmihalyi, M. (1990). Flow: The Psychology of Optimal Experience. New York: Harper, 1990.

4. Good, L. and Bederson, B. B. (2002). Zoomable User Interfaces as a Medium for Slide Show Presentations. Information Visualization, 2(2).

5. Marshall, C. C. and Shipman, F. M. (1994). VIKI: Spatial Hypertext Supporting Emergent Structure. Proceedings of 1994 ACM European Conference on Hypermedia Technology. pp 13-23.

6. Microsoft PowerPoint. (2001). http://www.microsoft.com/powerpoint/. 